\title{
Autonomous Homing and Docking Tasks for an Underwater Vehicle
}

\author{
Pakpong Jantapremjit ${ }^{1}$, Philip A. Wilson ${ }^{2}$ \\ ${ }^{1}$ Department of Mechanical Engineering, Faculty of Engineering \\ Burapha University \\ Bangsaen Campus, Chonburi, Thailand \\ E-mail: 1pakpong9@soton.ac.uk,pakpong@buu.ac.th \\ ${ }^{2}$ Fluid-Structure Interactions Research Group, School of Engineering Sciences \\ University of Southampton \\ Highfield, Southampton, United Kingdom \\ Tel: +44 (0)23 8059 6619, Fax: +44 (0)23 80595167 \\ E-mail: ${ }^{2}$ Philip.Wilson@soton.ac.uk
}

\begin{abstract}
This paper briefly introduces a strategy for autonomous homing and docking tasks using an autonomous underwater vehicle. The control and guidance based path following for those tasks are described in this work. A standard sliding mode for controller design is briefly given. The method provides robust motion control efforts for an underwater vehicle's decoupled system whilst minimising chattering effects. In a guidance system, the vector field based on a conventional artificial potential field method gives a desired trajectory with a use of existing information from sensors in the network. A well structured Line-of-Sight method is used for an AUV to follow the path. It provides guidance for an AUV to follow the predefined trajectory to a required position with the final desired orientation at the dock. Integration of a control and guidance system provides a complete system for this application. Simulation studies are illustrated in the paper.
\end{abstract}

\section{Keywords:}

Autonomous homing and docking, AUV, sliding mode control, vector field, line-of-sight

\section{Introduction}

Autonomous Underwater Vehicles (AUVs) are highly efficient which allows for ability to take control actions more accurately and reliably without human intervention. Long-term applications include collecting biological and mineral resources, seabed mapping and surveying, studying underwater and under-ice environments [1]. The power and data storage for current AUV designs do not allow for longterm deployments. Battery-powered can only rely upon for a few hours before power is depleted. In these long-term missions, data storage are also limited. It is therefore obvious that power and data storage are critical factors for all long-term operations. The inherent short operational periods limit the scope of each undersea exploration. In the future applications, an underwater vehicle should be able to operate continuously to complete one or many large mission(s). However, most underwater vehicles are typically only configured of short-term operation. Vehicles require both software and hardware to be turned off before its batteries can be manually recharged or replaced. To overcome the limitations of the onboard battery and data transfer procedure, a floating docking platform is required to extend the scope of potential missions. A focus on homing and docking operations allows a vehicle to come back to the dock to recharge its own battery and exchange data before continuing its operation. To be able to perform its homing and docking mission accurately, the guidance, navigation and control system must be reliable.

This work aims to develop a strategy in the control and guidance system required for a homing and docking strategy for an AUV. A model of an AUV using this strategy is briefly described and used throughout the paper. Homing and docking strategies are detailed. A trajectory planning for a homing and docking problem is formulated. For an assumption used in this paper, the AUV which is equipped with sensor units is able to track position and orientation from the existing sensor networks technology such as a long baseline system in the environment. In the homing stage, the conventional artificial potential field provides the obstacle avoidance. In the docking stage, the vector field provides guidance for a virtual AUV to follow the path to a required position with a final desired orientation. A weighted vector field is a better model to enhance performance in achieving the desired target. The Line-of-Sight technique allows an AUV to follow the predefined path to the dock. For control system design, a standard sliding mode control is presented. The controller utilises sliding surfaces based in time. A discussion on how this controller can be used for tracking errors of desired states is given.

The paper is organised as follows: Section 2 provides a kinematic model of an AUV. The control law using a sling mode control is detailed in section 3. Section 4 presents a guidance law based on the Line-of-Sight and the vector field path generation. The homing and docking strategy are given in section 5 . Integration system with simulation studies for a guidance and control is presented in section 6 . Finally, section 7 gives a conclusion. 


\section{AUV Modelling}

Attitude representation of the kinematic AUV model in the global reference frame is defined using Euler angles [2]. The kinematic equation is therefore written as,

$$
\dot{\eta}=J(\Theta) v=\left[\begin{array}{cc}
R(\Theta) & 0_{3 \times 3} \\
0_{3 \times 3} & T(\Theta)
\end{array}\right]\left[\begin{array}{c}
v_{1} \\
v_{2}
\end{array}\right],
$$

where $R(\Theta), T(\Theta) \in \mathfrak{R}^{3 \times 3}$ and $\eta, v \in \mathfrak{R}^{6 \times 1}$,

and,

$$
\begin{aligned}
& v=[u, v, w, p, q, r]^{T}, \\
& \eta=[x, y, z, \phi, \theta, \varphi]^{T},
\end{aligned}
$$

where $[u, v, w]^{T},[p, q, r]^{T}$ are linear and angular velocities in the $x-y-z$ directions and $[x, y, z]^{T}$ is the position in the $x-$ $\mathrm{y}-\mathrm{z}$ directions, $[\phi, \theta, \psi]^{T}$ is the Euler angles parameters (see Figure 1).

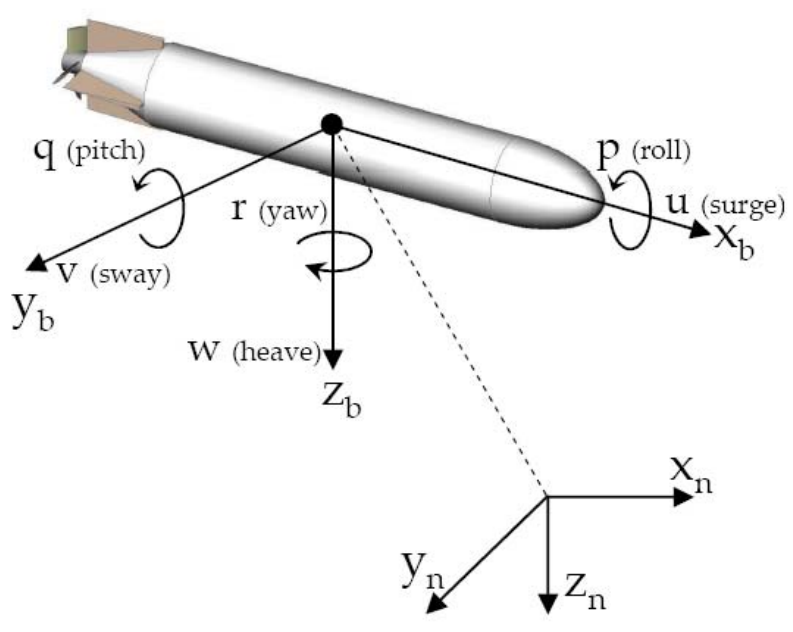

Figure 1 - Six degrees of freedom of an AUV in surge, sway, heave, roll, pitch and yaw motion

\section{Control}

Stability schemes in attitude and depth control for nonlinear system [3] are formulated based on the Lyapunov method. The method is a powerful tool for stability analysis which can be used for the design in various nonlinear controllers. One of the most common nonlinear feedback controller designs based on the Lyapunov analysis is the sliding mode control (SMC). It is categorised as a variable structure control system [4]. SMC has been used for AUV control because of excellent stability, robustness and disturbance rejection characteristics. Fundamentally, the sliding mode controller is composed of two main parts, namely the nominal part and also discontinuous terms dealing with uncertainties [5]. The controller with the typical sliding mode drives the system state trajectory onto the sliding surface and maintains that trajectory onto the surface for all times. Thus, the sliding mode becomes insensitive to system disturbances whilst on the sliding surface. Furthermore the significant characteristics of the sliding mode are order reduction and robust stability [5].

\section{Sliding Surface}

The sliding surface is designed so that the surface tends to and converges to zero when it satisfies the Lyapunov stability criterion thus the problem of tracking denoted as $x \equiv x_{d}$ is equivalent to that of remaining on the sliding surface for all time $\mathrm{t}>0$. The detail of a time-varying sliding surface $\sigma$ can be found in [6]. The control inputs can be regarded as that for the nominal plant and for the uncertainty of model parameter,

$$
u=-k x-K \operatorname{sgn}(\sigma)
$$

where $K$ is a constant, corresponding to the maximum value of the controller output. Reducing chattering which is caused by a signum function, a thin boundary layer of thickness around the switching surface is proposed [6],

$$
u=-k x-K \operatorname{sat}\left(\frac{s}{\Phi}\right)
$$

where the constant $\Phi$ defines the thickness of the boundary layer and $\operatorname{sat}\left(\frac{\sigma}{\Phi}\right)$ is a saturation function that is defined as,

$$
\operatorname{sat}(\sigma)=\left\{\begin{array}{cc}
\frac{\sigma}{\Phi}, & \text { if }\left|\frac{\sigma}{\Phi}\right| \leq 1 ; \\
\operatorname{sgn}\left(\frac{\sigma}{\Phi}\right), & \text { otherwise }
\end{array}\right.
$$

\section{Subsystems}

A controller designed for an AUV control needs to be robust to deal with external disturbance and model uncertainties. However, a simple model is required thus computational time will be relatively short. A controller that is decoupled into two subsystems of heading and depth (see Figure 2) is proposed [6]. An extension version for speed control subsystem is proposed in this work. The sliding mode control law are chosen as,

$$
\begin{gathered}
\bar{u}_{u}=-k_{u}\left(u-u_{d}\right)+\dot{u}_{d} \\
\bar{u}_{\varphi}=\varphi-k_{\varphi}(u \sin \theta \cos \varphi)-K s a t\left(\frac{\tilde{\varphi}}{\phi_{\varphi}}\right) \\
\bar{u}_{\theta}=\theta-k_{\theta}(u \sin \theta)-K s a t\left(\frac{\tilde{\theta}}{\phi_{\theta}}\right)
\end{gathered}
$$

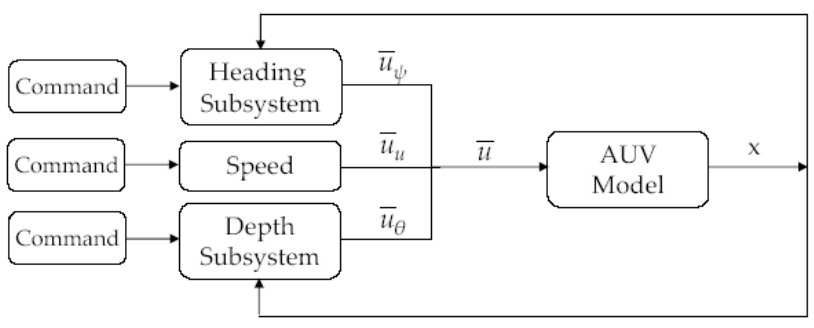

Figure 2 - Subsystems 


\section{Guidance}

Various guidance strategies are considered in the communities. A unified control based LOS concept [7] has been proposed which inspires this work. Path following techniques with an optimal cross-track controller [8] is considered for minimising the error. Inspired by various literatures, a guidance based control for following the path defined in this section proposed.

The aim of the path following method proposed in this work is to allow an AUV to follow a predefined path which is represented by a series of vehicle's coordinates joined by line segments. A number of techniques have been developed to solve this problem. The Line-of-Sight (LOS) guidance technique is intuitive and widely used in the application for path following of an underwater vehicle both in 2D and 3D. Line-of-sight guidance can be characterised by,

$$
\begin{aligned}
& \varphi_{d}=\arctan \left(\frac{-e_{y}}{\Delta}\right), \\
& \theta_{d}=\arctan \left(\frac{-e_{z}}{\sqrt{e_{y}^{2}+\Delta^{2}}}\right),
\end{aligned}
$$

where $\Delta$ is a look-ahead distance and $e_{y}, e_{z}$ are position along $\mathrm{y}$ and $\mathrm{z}$ axes, respectively. With the control laws developed using the sliding mode control developed in Section 3, the goal of the path following using LOS is ensured that,

$$
\begin{aligned}
& \tilde{u}=u-u_{d} \rightarrow 0, \\
& \tilde{\theta}=\theta-\theta_{d} \rightarrow 0, \\
& \tilde{\varphi}=\varphi-\varphi_{d} \rightarrow 0,
\end{aligned}
$$

\section{Homing and Docking Strategy}

In a long-term underwater application, a docking operation is required for recharging power and transferring data. However it is limited by the on-board energy capacity and data storage. A home returning and docking task is required during the transit of the mission. Therefore, position and orientation of AUV near a docking platform are accurately required. In the following sections, homing and docking are detailed.

\section{Homing Strategy}

A homing and docking strategy is now presented. A simple diagram depicted in Figure 3 shows how an AUV performs either the homing or the docking strategy [9]. Firstly, an AUV is located far away from a docking station where the distance between an AUV and the station must satisfy $|q|>2 D$, where $D$ is a radius between the dock $N_{1}$ and the sensor network $N_{2}, N_{3}$. With this condition an AUV should be able to do the homing strategy $L_{H}$ by using an artificial potential field path generation method. Secondly, when $|q| \leq 2 D$, it is possible for an AUV to perform docking preparation strategy $L_{D}$. The distance $2 D$ should allow an AUV enough distance and time to permit the desired position and orientation at the dock to be achieved. The homing and docking strategies are detailed in the following sections.

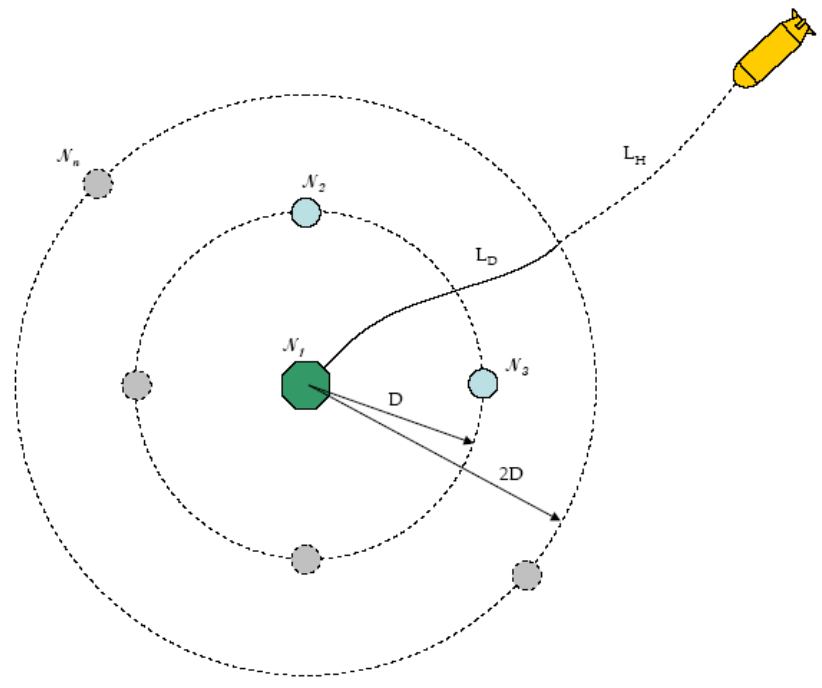

Figure 3 - Homing and docking path

Samples of $2 D$ home planning using a conventional potential field method are shown in Figure 4.

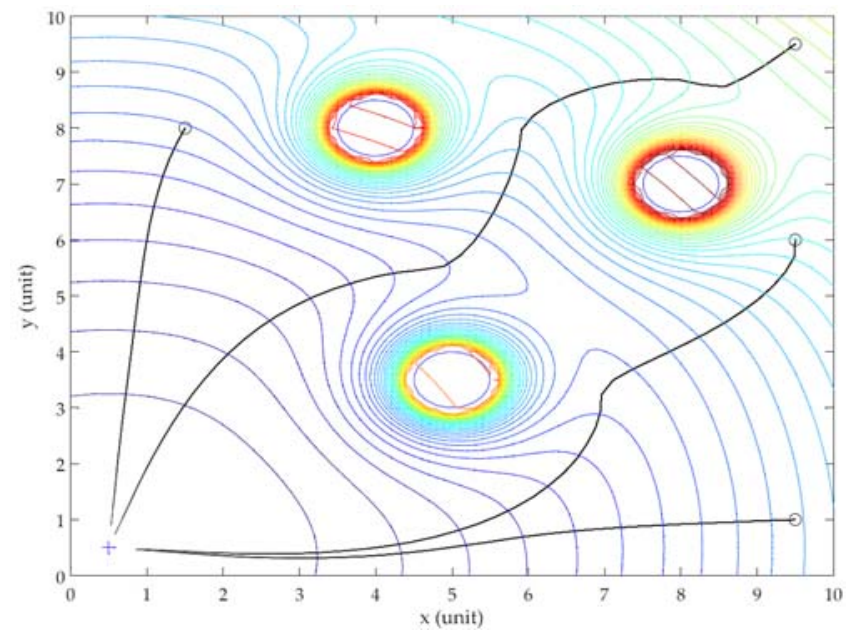

Figure 4 - Simulation results of home planning paths using a potential field method with three obstacles 


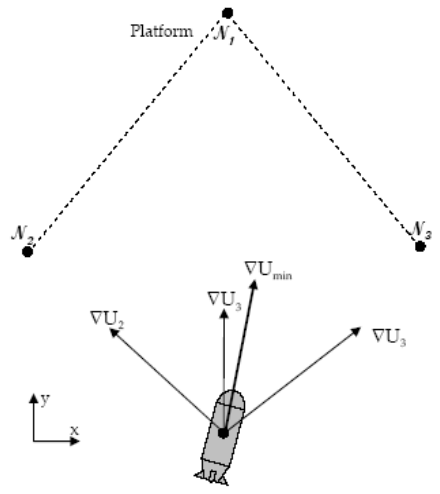

(a)

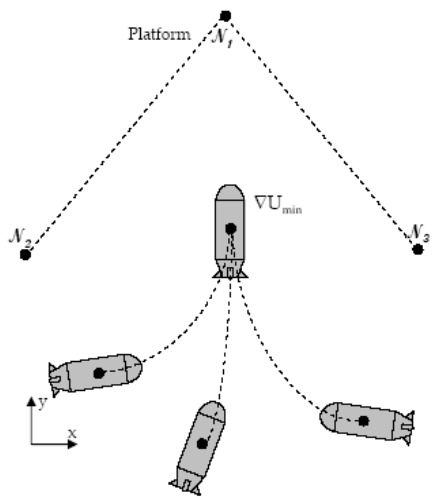

(b)

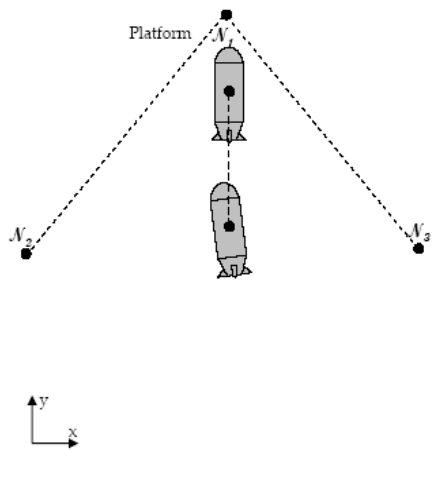

(c)

Figure 5 - An AUV is influenced by three sensors. Sensors are represented by $\bullet$ (a) Summing all vector fields, AUV finds the minimum potential force (b) AUV visits a valley which is around the centre area in the convex hull (c) Whilst approaching close to the target AUV is performing docking at the docking station

\section{Docking Strategy}

The docking strategy is more challenging than the homing task. Generally the docking strategy is divided into two stages: docking preparation and final docking. In the first stage, a precise tracking is used for relative position and motion between an AUV and platform. In the second stage, the docking velocity is kept within a safe range in order to avoid a possible serious impact between an AUV and the platform. A visual docking is a possible method that can be used to perform the final docking. However, this paper does not address the final docking manoeuvre. The docking preparation is considered by using the path generation method. This is similar to a method derived from the visual based navigation of insects [10], an average landmark vector model [11], a modified technique for a potential field method, also called a vector field, and a switching weighted vector field. In this paper, a vector field method aims to generate a predefined path in the docking trajectory. The compact representation is introduced,

$$
\nabla U(q)=-\sum_{i=1}^{n} \frac{N_{i}(q)-N(q)}{\left\|N_{i}(q)-N(q)\right\|}
$$

where $q=[x, y]^{T} \in \mathfrak{R}^{2}$ or $q=[x, y, z]^{T} \in \mathfrak{R}^{3}$ is a unit length point towards a sensor in the network. Later it is more convenient to compute the minimum potential force as,

$$
\nabla U_{\min }=\min (\nabla U(q))
$$

Where $\min (\nabla U(q))$ returns the minimum vector among all $\nabla U$. Minimising $\nabla U_{\text {min }}$ gives the goal of its minimum potentials which is close to the target point. The minimization point of the sum of potentials of each node will fall down to the valley area with respect to level (see equation (7)) and then converges to a minimum. The control algorithm starts determining the gradient of the potential functions related to all three sensor nodes as shown in Figure 5(a) leading a trajectory of a vehicle approaching the target along the gradient flows towards a single minimum potential. Figure 5(b) shows that a robot approaching a valley where its potential field converges towards the minimum. Then, the algorithm will lead a vehicle to achieve both position and orientation along the computed trajectory resulting in a docking manoeuvre at docking station as shown in Figure 5(c). Figure 6 shows a simulation studied for path generation during the docking preparation stage.

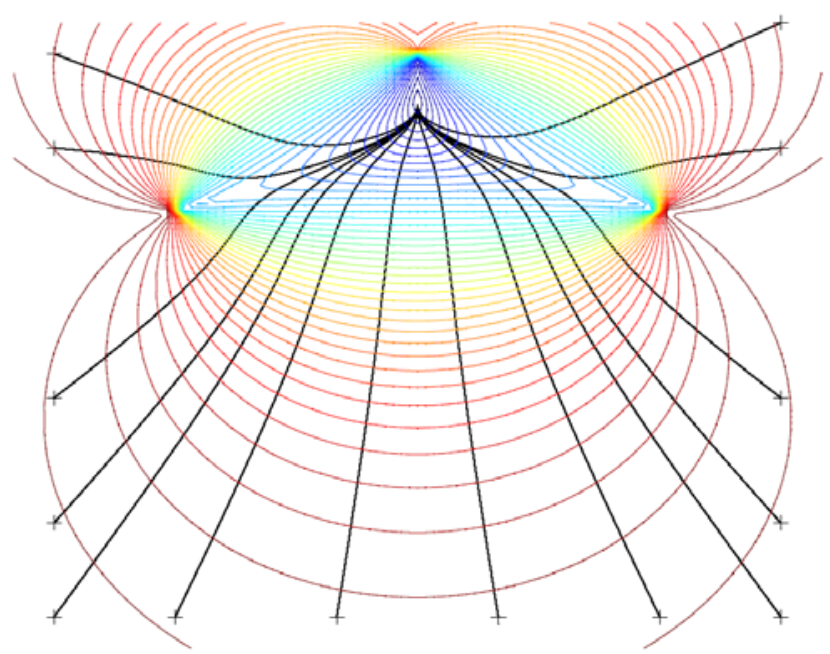

Figure 6 - Path planning for docking preparation

\section{Guidance and Control}

For the design of the entire system, an integration of control and guidance is introduced. As shown in Figure 2, a simple block diagram for guidance system is considered. The predefine path generated by the vector field method (see Section 4) is now available for the guidance system. Using the Line-of-Sight method, an AUV is able to follow the predefined path. The control system is designed using sliding mode control and provides the system stability such that it is guarantees that an AUV is able to converge to a desired path. 
In this preliminary result, a sliding mode is used for the heading controller which allows an AUV to track and converge to the path whilst the random noise is modelled for a disturbance in each state. Figure 7 and 8 show examples of the simulation study that shows an AUV following a predefined path in 2D and 3D with a constant speed of $1.3 \mathrm{~m} / \mathrm{s}$.

\section{Conclusion}

A strategy of a control and guidance system for an AUV's docking and homing tasks is developed in this work. A standard sliding mode controller was introduced, and the method minimises the chattering effects. For a guidance system, a predefined trajectory for homing and docking using a vector field method based on the potential field motion planning is presented. Using the Line-of-Sight guidance and sliding mode controller, it is shown that a vehicle is able to converge and follow the predefined path and match both position and orientation at the dock as demonstrated with the simulations.

\section{References}

[1] Griffiths, G. Technology and Applications of Autonomous Underwater Vehicles. Taylor and Francis, London, UK, 2003.

[2] Fossen, T. Guidance and Control of Ocean Vehicles. John Wiley and Sons Ltd., England, 1994.
[3] Yuh, J. Design and control of autonomous underwater robots: A survey. Autonomous Robots, 8(24), 2000.

[4] Utkin, V. Sliding Modes and Their Application in Variable Structure Systems. MIR Publishers, Moscow, 1978.

[5] Slotine, J.; and Li W. Applied Nonlinear Control. Prentice-Hall, Inc, New Jersey, 1991.

[6] Healey, A.; and Lienard, D. Multivariable sliding mode control for autonomous diving and steering of unmanned underwater vehicles. IEEE Journal of Oceanic Engineering, 18(3):327-339, 1993.

[7] Breivik M.; and Fossen T. A unified control concept for autonomous underwater vehicles. In Proceedings of the 2006 American Control Conference, 4920-4926, 2006.

[8] Bøhaug E., Pettersen K.; and Pavlov A. An optimal guidance scheme for cross-track control of underactuated underwater vehicles. In Proceedings of 14th Mediterranean Conference on Control and Automation, 1-5, 2006.

[9] Jantapremjit, P.; Wilson, P.A.; Guidance-control based path following for homing and docking using an Autonomous Underwater Vehicle. In 2008 IEEE International Conference Oceans’08, 6pp, 2008.

[10] Moller, R. Insect visual homing strategies in a robot with analog processing. Biological Cybernetics, 231-243, 2000.

[11] Wei R.; Mahony R.; and Austin, D. A bearing-only control law for stable docking of unicycles. In IEEE/RSJ International Conference on Intelligent Robots and Systems, 27-31, 2003.

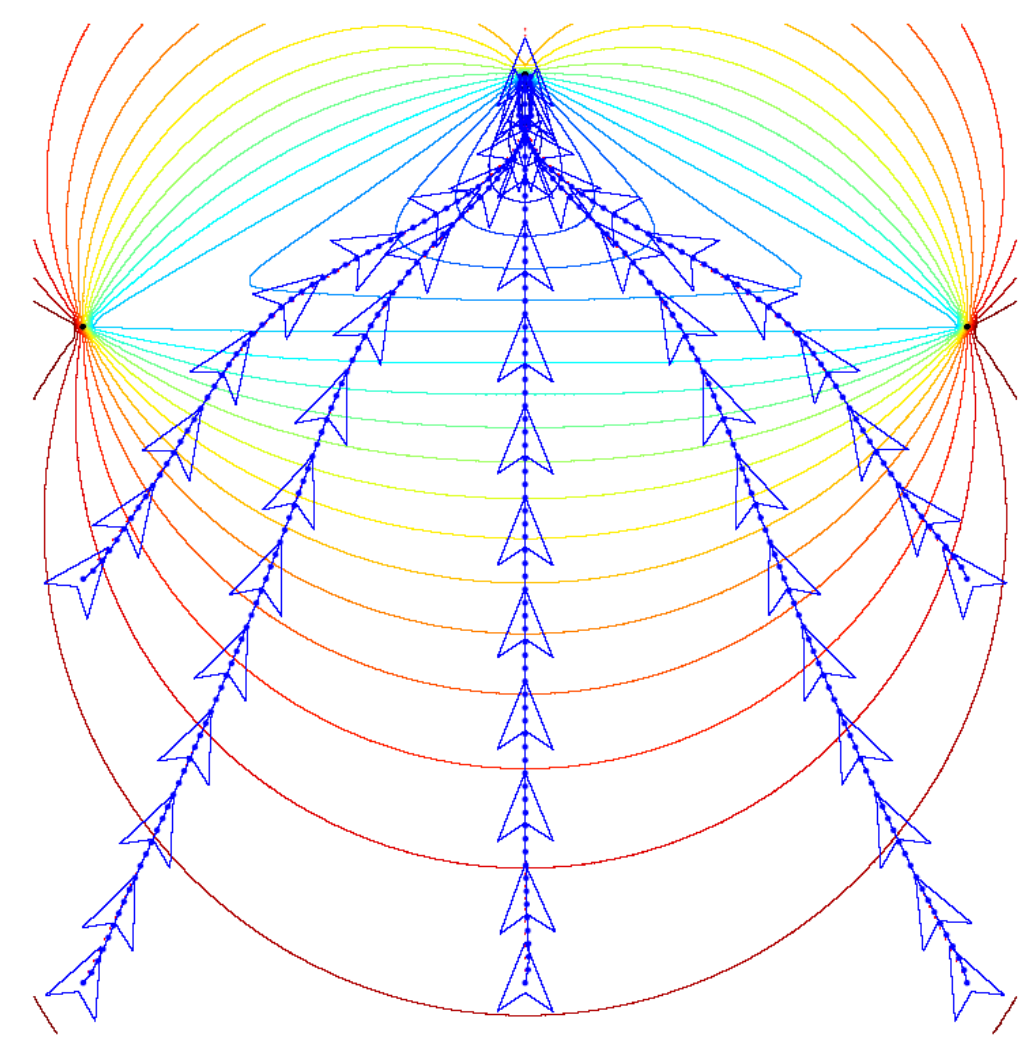

Figure 7 - Simulations show an AUV follows the path generated by vector field method in $2 D$ 


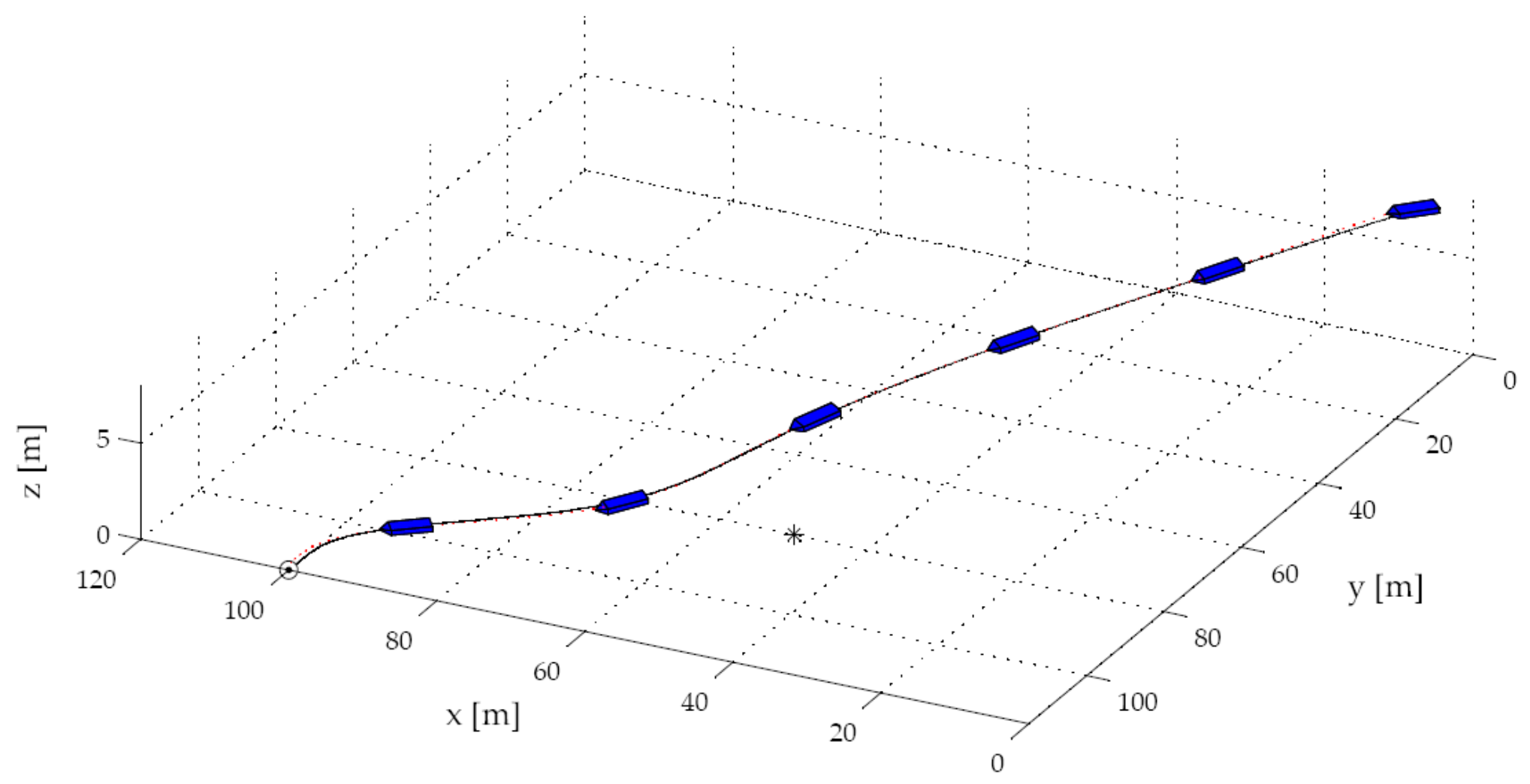

Figure 8 - Example of a simulation shows an AUV follows the path generated by vector field method in $3 D$ 